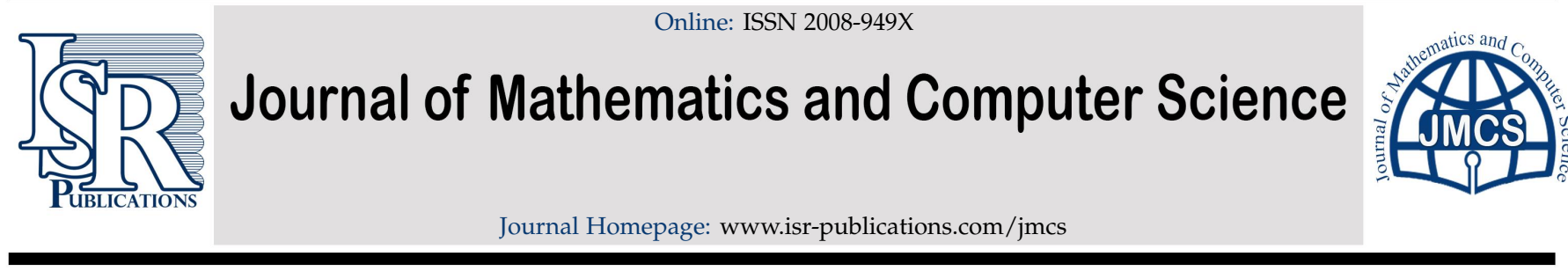

\title{
Positive solutions to a nonlinear eigenvalue problem
}

Yong-Hui Zhou

School of Mathematics and Statistics, HeXi University, Zhangye, Gansu 734000, P. R. China.

\begin{abstract}
In this paper, the existence of positive solutions to a nonlinear eigenvalue problem is obtained by Leray-Schauder fixed point theorem.
\end{abstract}

Keywords: Existence, positive solutions, Leray-Schauder fixed point theorem.

2020 MSC: 34B15.

(C)2020 All rights reserved.

\section{Introduction}

In this paper, we consider the nonlinear eigenvalue two-point boundary value problem

$$
\left\{\begin{array}{l}
u^{(4)}(t)=\lambda h(t) f(u(t)), \quad t \in(0,1) \\
u(0)=u^{\prime}(1)=u^{\prime \prime}(0)=u^{\prime \prime \prime}(1)=0
\end{array}\right.
$$

where $\lambda>0$ is a positive parameter.

We will make the following assumptions:

(i) $f:[0,1) \longrightarrow R$ is continuous and $f(0)>0$;

(ii) $h(t) \in C[0,1]$ and there exist two constants $\tau, k: \tau \in[0,1], k \in(1, \infty)$ such that $h(\tau) \neq 0$ and

$$
\int_{0}^{1} G(t, s) h^{+}(s) d s \geqslant k\left[\int_{0}^{1} G(t, s) h^{-}(s) d s\right]
$$

for $t \in[0,1]$, where $a^{+}$is the positive part of $a$ and $a^{-}$is the negative part of $a$.

Next, we state the main result.

Theorem 1.1. Let (i) and (ii) hold. Then there exists a positive number $\lambda^{*}$ such that BVP (1.1) has at least one positive solution for $\lambda: 0<\lambda<\lambda^{*}$.

Email address: 2823877618@qq. com (Yong-Hui Zhou)

doi: $10.22436 /$ jmcs.021.01.02

Received: 2019-09-11 Revised: 2020-02-06 Accepted: 2020-02-19 


\section{Preliminaries lemmas}

To prove Theorem 1.1, we need several preliminary results.

Lemma 2.1. For $\mathrm{y} \in \mathrm{C}[0,1]$, the problem

$$
\left\{\begin{array}{l}
u^{(4)}(t)=y(t) t \in(0,1) \\
u(0)=u^{\prime}(1)=0=u^{\prime \prime}(0)=u^{\prime \prime \prime}(1)=0
\end{array}\right.
$$

has a unique solution

$$
u(t)=\int_{0}^{1} G(t, s) y(s) d s
$$

where

$$
G(t, s)=\frac{1}{6} \begin{cases}\left(6 t-3 t^{2}-s^{2}\right) s, & 0 \leqslant s \leqslant t \leqslant 1 \\ \left(6 s-3 s^{2}-t^{2}\right) t, & 0 \leqslant t \leqslant s \leqslant 1\end{cases}
$$

Lemma 2.2. If $y \in C[0,1], y \geqslant 0$, then the unique solution $u$ of the (2.1) satisfies

$$
u \geqslant 0, t \in[0,1]
$$

Moreover, if $\mathrm{y}_{1}(\mathrm{t}) \geqslant \mathrm{y}_{2}(\mathrm{t})$ for $\mathrm{t} \in[0,1]$, then the corresponding solutions $\mathrm{u}_{1}(\mathrm{t})$ and $\mathrm{u}_{2}(\mathrm{t})$ satisfy

$$
u_{1}(t) \geqslant u_{2}(t), \text { for } t \in[0,1] \text {. }
$$

Lemma 2.3. Let (i) and (ii) hold, then for every $0<\delta<1$, there exists a positive number $\lambda_{1}$ such that, for $0<\lambda<\lambda_{1}$, the problem

$$
\left\{\begin{array}{l}
u^{(4)}(t)=\lambda h^{+}(t) f(u(t)), \quad t \in(0,1) \\
u(0)=u^{\prime}(1)=0=u^{\prime \prime}(0)=u^{\prime \prime \prime}(1)
\end{array}\right.
$$

has a positive solution $\mathfrak{u}_{\lambda_{1}}$ with $\left|\mathfrak{u}_{\lambda_{1}}\right|_{0} \longrightarrow 0$ as $\lambda \longrightarrow 0$, and

$$
u_{\lambda_{1}} \geqslant \lambda \delta f(0) p(t), t \in[0,1],
$$

where

$$
p(t)=\int_{0}^{1} G(t, s) h^{+}(s) d s .
$$

Proof. By Lemma 2.2, we know that $p(t) \geqslant 0$ for $t \in[0,1]$. From Lemma 2.1, (2.2) is equivalent to the integral equation

$$
u(t)=\lambda \int_{0}^{1} G(t, s) h^{+}(s) f u(s) d s:=A u(t),
$$

where $u \in C[0,1]$. Then $A: C[0,1] \longrightarrow C[0,1]$ is completely continuous and fixed points of $A$ are solutions of (2.2). We apply the Leray-Schauder fixed point theorem to prove $A$ has a fixed point.

Let $\varepsilon>0$ be such that

$$
f(t) \geqslant \delta f(0), \text { for } 0 \leqslant \varepsilon .
$$

Suppose that

$$
\lambda<\frac{\varepsilon}{2|p|_{0} f_{1}(\varepsilon)}:=\lambda_{1}
$$

where

$$
f_{1}(t)=\max _{s \in[0, t]} f(s) .
$$

Since

$$
\lim _{t \rightarrow 0^{+}} \frac{f_{1}(t)}{t}=+\infty,
$$


it follows that there exists $\tau_{\lambda} \in(0, \varepsilon)$, such that

$$
\frac{f_{1}\left(\tau_{\lambda}\right)}{\tau_{\lambda}}=\frac{1}{2 \lambda|p|_{0}}
$$

We note that (2.4) implies

$$
\tau_{\lambda} \longrightarrow 0 \text { as } \lambda \longrightarrow 0
$$

Now, we consider the equations

$$
u=\theta A u, \theta \in(0,1),
$$

let $u \in C(0,1)$ and $\theta \in(0,1)$ be such that $u=\theta A u$. We claim that $|u|_{0} \neq \tau_{\lambda}$. In fact

$$
u(t)=\theta \lambda \int_{0}^{1} G(t, s) h^{+}(s) f u(s) d s,
$$

set

$$
w(t)=\theta \lambda \int_{0}^{1} G(t, s) h^{+}(s) f_{1}|u|_{0} d s \leqslant \theta \lambda f_{1}\left(|u|_{0}\right) p(t),
$$

then by Lemma 2.2 and the fact that $f(u) \leqslant f_{1}\left(|u|_{0}\right)$, we know that

$$
u(t) \leqslant w(t), \text { for } t \in[0,1]
$$

Moreover, we have

$$
|\mathfrak{u}|_{0} \leqslant\left.\lambda|p|\right|_{0} f_{1}\left(|u|_{0}\right)
$$

or

$$
\frac{f_{1}\left(|u|_{0}\right)}{|u|_{0}} \geqslant \frac{1}{\lambda|p|_{0}}
$$

which implies that $|\mathfrak{u}|_{0} \neq \tau_{\lambda}$. Thus by Leray-Schauder fixed point theorem, $A$ has a fixed point $u_{\lambda_{1}}$ with

$$
\left|u_{\lambda_{1}}\right|_{0} \leqslant \tau_{\lambda}<\varepsilon
$$

Therefore, combining (2.3), (2.5), and using Lemma 2.2, we have that

$$
u_{\lambda_{1}}(t) \geqslant \lambda \delta f(0) p(t), t \in[0,1] .
$$

\section{Proof of the main result}

Proof of Theorem 1.1. Let

$$
q(t)=\int_{0}^{1} G(t, s) h^{-}(s) d s,
$$

then $q(t) \geqslant 0$. By (ii), there exist positive numbers $c \in(0,1), d \in(0,1)$ such that

$$
q(t)|f(y)| \leqslant d p(t) f(0)
$$

for $y \in[0, c]$ and $t \in[0,1]$. Fix $\delta \in(d, 1)$, and let $\lambda_{2}>0$ be such that

$$
\left|u_{\lambda_{1}}\right|_{0}+\lambda \delta f(0)|p|_{0} \leqslant c
$$

for $\lambda<\lambda_{2}$, where $u_{\lambda_{1}}$ is given by Lemma 2.3, and

$$
|f(x)-f(y)| \leqslant f(0)\left(\frac{\delta-d}{2}\right)
$$

for $x \in[-c, c], y \in[-c, c]$ with $|x-y| \leqslant \lambda_{2} \delta f(0)|p|_{0}$. 
Let $\lambda<\lambda_{2}$, we look for a solution $u_{\lambda}$ of the form $u_{\lambda}+v_{\lambda}$. Here $v_{\lambda}$ solves

$$
\left\{\begin{array}{l}
u^{(4)}(t)=\lambda h^{+}(t)\left(f\left(u_{\lambda_{1}}+v\right)-f\left(u_{\lambda_{1}}\right)\right)-\lambda h^{-}(t) f\left(u_{\lambda_{1}}+v\right), t \in(0,1) \\
u(0)=u^{\prime}(1)=0=u^{\prime \prime}(0)=u^{\prime \prime \prime}(1)=0
\end{array}\right.
$$

For each $\omega \in \mathrm{C}[0,1]$, let $v=\mathrm{T}(\omega)$ be the solution of

$$
\left\{\begin{array}{l}
u^{(4)}(t)=\lambda h^{+}(t)\left(f\left(u_{\lambda_{1}}+w\right)-f\left(u_{\lambda_{1}}\right)\right)-\lambda h^{-}(t) f\left(u_{\lambda_{1}}+w\right), t \in(0,1) \\
u(0)=u^{\prime}(1)=0=u^{\prime \prime}(0)=u^{\prime \prime \prime}(1)=0
\end{array}\right.
$$

then $\mathrm{T}: \mathrm{C}[\mathrm{O}, 1] \longrightarrow \mathrm{C}[0,1]$ is completely continuous. Let $v \in \mathrm{C}[0,1]$ and $\theta \in \mathrm{C}[0,1]$ be such that $v=\theta T v$. Then we have

$$
\left\{\begin{array}{l}
u^{(4)}(t)=\theta \lambda h^{+}(t)\left(f\left(u_{\lambda_{1}}+v\right)-f\left(u_{\lambda_{1}}\right)\right)-\theta \lambda h^{-}(t) f\left(u_{\lambda_{1}}+v\right), t \in(0,1) \\
u(0)=u^{\prime}(1)=0=u^{\prime \prime}(0)=u^{\prime \prime \prime}(1)=0
\end{array}\right.
$$

We claim that $|v|_{0} \neq \lambda \delta f(0)\left|p_{0}\right|$. Suppose to the contrary that $|v|_{0} \neq \delta f(0)\left|p_{0}\right|$. Then by (3.2) and (3.3), we obtain

$$
\left|u_{\lambda_{1}}+v\right|_{0} \leqslant\left.\left|u_{\lambda_{1}}\right|_{0}|+| v\right|_{0} \leqslant c
$$

and

$$
\left|f\left(u_{\lambda_{1}}+v\right)-f\left(u_{\lambda_{1}}\right)\right|_{0} \leqslant f(0)\left(\frac{\delta-d}{2}\right) .
$$

Using (3.1), (3.4), Lemmas 2.1, and 2.2, we have

$$
|v(t)| \leqslant \lambda\left(\frac{\delta-d}{2}\right) \mathbf{f}(0) p(t)+\lambda d f(0) p(t)=\lambda\left(\frac{\delta+d}{2}\right) f(0) p(t) .
$$

In particular,

$$
|v|_{0} \leqslant \lambda\left(\frac{\delta+d}{2}\right) f(0) p_{0}<\lambda \delta f(0)|p|_{0}
$$

is a contradiction, and the claim is proved. Thus by Leray-Schauder fixed point theorem, $\mathrm{T}$ has a fixed point $v_{\lambda}$ with

$$
\left|v_{\lambda}\right|_{0} \leqslant \lambda \delta f(0)|p|_{0}
$$

Using (2.2) and (3.5), we obtain

$$
u_{\lambda} \geqslant u_{\lambda_{1}}-\left|v_{\lambda}\right| \geqslant \lambda \delta f(0) p(t)-\lambda\left(\frac{\delta+d}{2}\right) f(0) p(t)
$$

and

$$
\lambda \delta f(0) p(t)-\lambda\left(\frac{\delta+d}{2}\right) f(0) p(t)=\lambda\left(\frac{\delta-d}{2}\right) f(0) p(t) .
$$

Therefore,

$$
u_{\lambda} \geqslant \lambda\left(\frac{\delta-d}{2}\right) f(0) p(t) \geqslant 0,
$$

i.e., $u_{\lambda}$ is a positive solution of (1.1). The proof is completed.

\section{Acknowledgment}

This paper is supported by the Youth Fund of Hexi University of China (QN2018008).

\section{References}

[1] R. I. Avery, A generalization of the Leggett-Williams fixed point theorem, Math. Sci. Res. Hot-line., 3 (1998), 9-14.

[2] R. I. Avery, A. C. Peterson, Three positive fixed points of nonlinear operators on ordered Banach spaces, Comput. Math. Appl., 42 (2001), 313-322. 
[3] Z. B. Bai, The upper and lower solution method for some fourth-order boundary value problems, Nonlinear Anal., 67 (2007), 1704-1709.

[4] C. Z. Bai, Triple positive solutions of three-point boundary value problems of fourth-order differential equations, Comput. Math. Appl., 56 (2008), 1364-1371.

[5] Z. B. Bai, Y. F. Wang, W. G. Ge, Triple positive solutions for a class of two-point boundary value problems, Electron. J. Differential Equations, 2004 (2004), 8 pages.

[6] J. R. Graef, L. J. Kong, B. Yang, Positive solutions of boundary value problems for discrete and continuous beam equations, J. Appl. Math. Comput., 41 (2013), 197-208.

[7] B. Liu, Positive solutions of fourth-order two-point boundary value problems, Appl. Math. Comput., 148 (2004), $407-420$.

[8] J.-P. Sun, W.-T. Li, Y.-H. Zhao, Three positive solutions of a nonlinear three-point boundary value problem, J. Math. Anal. Appl., 288 (2003), 708-716.

[9] Y. P. Sun, X. P. Zhang, M. Zhao, Successive iteration of positive solutions for fourth-order two-point boundary value problems, Abstr. Appl. Anal., 2013 (2013), 8 pages.

[10] Y.-R. Yang, Triple positive solutions of a class of fourth-order two-point boundary value problems, Appl. Math. Lett., 23 (2010), 366-370. 